\title{
Monte Carlo approach to M-theory
}

\author{
Werner Krauth ${ }^{\mathrm{a}, 1}$, Hermann Nicolai ${ }^{\text {b,2,3 }}$, Matthias Staudacher ${ }^{\mathrm{b}, 4}$ \\ a CNRS-Laboratoire de Physique Statistique, Ecole Normale Supérieure 24, rue Lhomond, F-75231 Paris Cedex 05, France \\ b Albert-Einstein-Institut, Max-Planck-Institut für Gravitationsphysik, Schlaatzweg 1, D-14473 Potsdam, Germany
}

Received 24 March 1998

Editor: L. Alvarez-Gaumé

\begin{abstract}
We discuss supersymmetric Yang-Mills theory dimensionally reduced to zero dimensions and evaluate the $S U(2)$ and $S U(3)$ partition functions by Monte Carlo methods. The exactly known $S U(2)$ results are reproduced to very high precision. Our calculations for $S U(3)$ agree closely with an extension of a conjecture due to Green and Gutperle concerning the exact value of the $S U(N)$ partition functions. (C) 1998 Published by Elsevier Science B.V. All rights reserved.
\end{abstract}

\section{The model}

Recently there has been renewed interest in dimensionally reduced supersymmetric Yang-Mills theories. It has been demonstrated that reductions of the ten-dimensional theory to $p+1$ dimensions are relevant to the description of $p$-dimensional extended objects (D-branes) in string theory, and therefore, potentially, to quantum gravity. Furthermore, various such reductions are currently being investigated in attempts to find a theory, termed M-theory, which, it is hoped, non-perturbatively encompasses all known superstring models as well as $D=11$ supergravity. The most extreme such reduction, to zero dimensions, is believed to describe string instantons, i.e. configurations of open superstrings whose ends are fixed in space-time. The functional integral of Yang-Mills theory becomes an ordinary multi-dimensional integral, which turns out to be well-defined and finite if supersymmetry is present. The same integral appears in attempts to rigorously define and calculate the Witten index of quantum-mechanical supersymmetric gauge theory [1,2]. Lastly, it is the cornerstone of the

\footnotetext{
${ }^{1}$ E-mail: krauth@physique.ens.fr.

${ }^{2}$ E-mail: nicolai@aei-potsdam.mpg.de.

${ }^{3}$ Supported in part by EU Contract FMRX-CT96-0012.

${ }^{4}$ E-mail: matthias@aei-potsdam.mpg.de.
} 
so-called IKKT model [3] which is claimed to yield a non-perturbative definition of superstring theory. Explicitly, the integral reads, for gauge group $S U(N)$,

$$
\mathscr{Z}_{D, N}:=\int \prod_{A=1}^{N^{2}-1}\left(\prod_{\mu=1}^{D} \frac{d X_{\mu}^{A}}{\sqrt{2 \pi}}\right)\left(\prod_{\alpha=1}^{\mathscr{N}} d \Psi_{\alpha}^{A}\right) \exp \left[\frac{1}{2} \operatorname{Tr}\left[X_{\mu}, X_{\nu}\right]\left[X_{\mu}, X_{\nu}\right]+\operatorname{Tr} \Psi_{\alpha}\left[\Gamma_{\alpha \beta}^{\mu} X_{\mu}, \Psi_{\beta}\right]\right] .
$$

We do not include a coupling constant into this definition, since it could be immediately scaled out in a trivial fashion. The matrices in the exponent in Eq. (1) are in the fundamental representation of $S U(N)$, i.e. $X_{\mu}=X_{\mu}^{A} T_{A}, \Psi_{\alpha}=\Psi_{\alpha}^{A} T_{A}$, where the $S U(N)$ generators $T_{A}$ are hermitean and normalized such that $\operatorname{Tr} T^{A} T^{B}$ $=\frac{1}{2} \delta^{A B}$. The symmetric $\mathscr{N} \times \mathscr{N}$ matrices $\Gamma^{\mu}$ are related to the standard $S O(1, D-1)$ gamma matrices by $\Gamma^{\mu}=\mathscr{C} \gamma^{\mu}$, where $\mathscr{C}$ is the charge conjugation matrix. The model is supersymmetric in dimensions $D=3,4,6,10$, where the degree $\mathscr{N}$ of (real) supersymmetry is, respectively, $\mathscr{N}=2(D-2)=2,4,8,16$, with the supersymmetry variations

$$
\delta X_{\mu}=i \bar{\varepsilon} \gamma^{\mu} \Psi, \quad \delta \Psi=-\frac{i}{2}\left[X_{\mu}, X_{\nu}\right] \gamma^{\mu} \gamma^{\nu} \varepsilon
$$

The most interesting case, in view of the above applications, is the maximally supersymmetric integral with $D=10$.

We note that all calculations are performed with a Euclidean signature. The Wick rotation to Euclidean space is simply accomplished by the substitution $X_{0} \rightarrow i X_{D}$. This leads to a positive definite bosonic "action" in the exponent, which is a necessary (but not sufficient) prerequisite for the integral to be well-defined. When switching from Minkowskian to a Euclidean signature, one in principle faces the problem of fermion doubling because e.g. the Majorana-Weyl spinors required for maximally supersymmetric Yang-Mills theory exist only for signature $(1,9)$. However, this problem is spurious and completely resolved by working only with the Euclidean Majorana field $\Psi$, and never making use of its complex conjugate field [4]. This is consistent because the relevant requirement in Euclidean quantum field theory is not hermiticity, but rather Osterwalder-Schrader reflection positivity.

Integrating out the real Grassmann variables $\Psi_{\alpha}^{A}$, one obtains

$$
\mathscr{Z}_{D, N}=\int \prod_{A=1}^{N^{2}-1} \prod_{\mu=1}^{D} \frac{d X_{\mu}^{A}}{\sqrt{2 \pi}} \exp \left[\frac{1}{2} \operatorname{Tr}\left[X_{\mu}, X_{\nu}\right]\left[X_{\mu}, X_{\nu}\right]\right] \mathscr{P}_{D, N}(X)
$$

where $\mathscr{P}_{D, N}$ is a homogeneous polynomial of degree $k=(D-2)\left(N^{2}-1\right)=\frac{1}{2} \mathscr{N}\left(N^{2}-1\right)$ in the variables $X_{\mu}^{A}$. Its crucial property is invariance under $S O(D) \times S U(N)$. This polynomial is just the Pfaffian of the $2 k \times 2 k$ antisymmetric matrix

$$
\left(\mathscr{M}_{D, N}\right)_{\alpha \beta}^{A B}=-i f^{A B C} \Gamma_{\alpha \beta}^{\mu} X_{\mu}^{C},
$$

which can be directly read off from Eq. (1). Here $f^{A B C}=-2 i \operatorname{Tr} T^{A}\left[T^{B}, T^{C}\right]$ are the $S U(N)$ structure constants. The Pfaffian is explicitly given by

$$
\mathscr{P}_{D, N}=\operatorname{Pf} \mathscr{M}_{D, N}=\frac{1}{2^{k} k !} \sum_{\sigma}(-1)^{\sigma} \mathscr{M}_{D, N}^{\sigma_{1} \sigma_{2}} \ldots \mathscr{M}_{D, N}^{\sigma_{2 k-1} \sigma_{2 k}}
$$

Here $\sigma$ runs over all permutations of the $2 k$ double indices $\sigma \equiv(\alpha A)$, and $(-1)^{\sigma}$ is the sign of the permutation. Although the matrices $\mathscr{M}_{D, N}$ are complex in general, the Pfaffians are real as a consequence of the fact that we started from a hermitean action (in Minkowski signature). However, they are not necessarily positive. The explicit form of $\mathscr{M}_{D, N}$ depends on the particular representation of the Clifford algebra and the 
corresponding gamma matrices $\Gamma_{\mu}$. For definiteness, let us specify a convenient choice for the four dimensions of interest. Denote by $\boldsymbol{X}_{\boldsymbol{\mu}}$ the adjoint representation of $S U(N)$, i.e. $\boldsymbol{X}_{\mu}^{A B}=f^{A B C} X_{\mu}^{C}$.

$D=3$ : Here the Pfaffian is determined from the $2\left(N^{2}-1\right) \times 2\left(N^{2}-1\right)$ matrix

$$
\mathscr{M}_{3, N}(X)=\left(\begin{array}{cc}
\boldsymbol{X}_{3}+i \boldsymbol{X}_{2} & i \boldsymbol{X}_{1} \\
i \boldsymbol{X}_{1} & \boldsymbol{X}_{3}-i \boldsymbol{X}_{2}
\end{array}\right)
$$

$D=4$ : In this case it is possible to reduce to a complex two-dimensional representation instead of the real four-dimensional representation. This allows us to rewrite the Pfaffian as an ordinary determinant: there exists a $2\left(N^{2}-1\right) \times 2\left(N^{2}-1\right)$ matrix $\mathscr{M}_{4, N}^{\prime}$ such that Pf $\mathscr{M}_{4, N}=\operatorname{det} \mathscr{M}_{4, N}^{\prime}$, with

$$
\mathscr{M}_{4, N}^{\prime}(X)=\left(\begin{array}{ll}
\boldsymbol{X}_{4}+i \boldsymbol{X}_{3} & i \boldsymbol{X}_{2}+\boldsymbol{X}_{1} \\
i \boldsymbol{X}_{2}-\boldsymbol{X}_{1} & \boldsymbol{X}_{4}-i \boldsymbol{X}_{3}
\end{array}\right)
$$

$D=6$ : Again it is possible to replace the real eight-dimensional by a complex four-dimensional representation: we have $\operatorname{Pf} \mathscr{M}_{6, N}=\operatorname{det} \mathscr{M}_{6, N}^{\prime}$ with the $4\left(N^{2}-1\right) \times 4\left(N^{2}-1\right)$ matrix $\mathscr{M}_{6, N}^{\prime}$

$$
\mathscr{M}_{6, N}^{\prime}(X)=\left(\begin{array}{cc}
\boldsymbol{X}_{6}+i \boldsymbol{X}_{5} & i \boldsymbol{X}_{4}+\sigma_{1} \boldsymbol{X}_{1}+\sigma_{2} \boldsymbol{X}_{2}+\sigma_{3} \boldsymbol{X}_{3} \\
i \boldsymbol{X}_{4}-\sigma_{1} \boldsymbol{X}_{1}-\sigma_{2} \boldsymbol{X}_{2}-\sigma_{3} \boldsymbol{X}_{3} & \boldsymbol{X}_{6}-i \boldsymbol{X}_{5}
\end{array}\right),
$$

where the $\sigma_{i}$ are the standard Pauli matrices.

$D=10$ : In this case we use the decomposition

$$
\mathscr{M}_{10, N}(X)=\left(\begin{array}{cc}
\boldsymbol{X}_{10}+i \boldsymbol{X}_{9} & i \boldsymbol{X}_{8}+\Gamma^{i} \boldsymbol{X}_{i} \\
i \boldsymbol{X}_{8}-\Gamma^{i} \boldsymbol{X}_{i} & \boldsymbol{X}_{10}-i \boldsymbol{X}_{9}
\end{array}\right)
$$

for the $16\left(N^{2}-1\right) \times 16\left(N^{2}-1\right)$ matrix $\mathscr{M}_{10, N}$. The indices $i, j, k$ run from 1 to 7 , and the seven $8 \times 8$ matrices $\Gamma^{i}$ satisfy the $S O(7)$ Clifford algebra $\left\{\Gamma^{i}, \Gamma^{j}\right\}=2 \delta^{i j}$. A particularly nice representation is in terms of the octonionic structure constants $c_{i j k}$ (again with $i, j, k=1, \ldots, 7$ )

$$
\left(\Gamma^{i}\right)_{j k}=i c_{i j k}, \quad\left(\Gamma^{i}\right)_{88}=0, \quad\left(\Gamma^{i}\right)_{j 8}=-\left(\Gamma^{i}\right)_{8 j}=i \delta_{j}^{i}
$$

These structure constants are completely antisymmetric; the non-vanishing ones are fully specified by:

$$
c_{124}=c_{235}=c_{346}=c_{457}=c_{561}=c_{672}=c_{713}=1 .
$$

The $\Gamma^{i}$ are obviously hermitean and purely imaginary. Note that, unlike for $D=4$ and $D=6$, it is generally not possible here to reduce the Pfaffian to a determinant. The only exceptions are $N=2$ and $N=3$, since in this case the number of dimensions $(=10)$ exceeds the dimension of the gauge group, and we can use $S O(10)$ invariance to set $X_{9}=X_{10}=0$. Then the Pfaffian becomes

$$
\left.\mathscr{P}_{10, N}(X)\right|_{X_{9}=X_{10}=0}=\operatorname{det}\left(i \boldsymbol{X}_{8}+\Gamma^{i} \boldsymbol{X}_{i}\right)
$$

This allows to integrate, for $S U(3)$ and $D=10$, over only eight instead of ten matrices, an observation that will 
prove useful in the numerical calculations below. After proper inclusion of the Fadeev-Popov determinant associated with this reduction, the integral becomes

$$
\mathscr{Z}_{10,3}=\frac{1}{8 !} \int \prod_{a, A=1}^{8} \frac{d X_{a}^{A}}{\sqrt{2 \pi}} \exp \left[\frac{1}{2} \operatorname{Tr} \sum_{a, b=1}^{8}\left[X_{a}, X_{b}\right]\left[X_{a}, X_{b}\right]\right]\left[\operatorname{det}_{1 \leq c, C \leq 8} X_{c}^{C}\right]^{2} \operatorname{det} \mathscr{M}^{\prime}(X),
$$

where $\mathscr{M}^{\prime}(X)=i \boldsymbol{X}_{8}+\Gamma^{i} \boldsymbol{X}_{i}$.

For general $D$ and $N$, the Pfaffian polynomials $\mathscr{P}_{D, N}$ have a very complicated structure about which little is known. These complications are not yet fully apparent for $S U(2)$, where the Pfaffians are still comparatively simple $[1,2,5]$ :

$$
\begin{aligned}
& \mathscr{P}_{3,2}(X)=\frac{1}{3} \epsilon^{A B C} \epsilon_{\mu \nu \rho} X_{\mu}^{A} X_{\nu}^{B} X_{\rho}^{C}=-\frac{2}{3} i \epsilon_{\mu \nu \rho} \operatorname{Tr} X_{\mu}\left[X_{\nu}, X_{\rho}\right], \\
& \mathscr{P}_{4,2}(X)=\frac{2}{3} \epsilon^{A B C} \epsilon^{A^{\prime} B^{\prime} C^{\prime}} X_{\mu}^{A} X_{\mu}^{A^{\prime}} X_{\nu}^{B} X_{\nu}^{B^{\prime}} X_{\rho}^{C} X_{\rho}^{C^{\prime}}=\frac{8}{3} \operatorname{Tr}\left[X_{\mu}, X_{\nu}\right]\left[X_{\nu}, X_{\rho}\right]\left[X_{\rho}, X_{\mu}\right], \\
& \mathscr{P}_{6,2}(X)=\left(\frac{8}{3} \operatorname{Tr}\left[X_{\mu}, X_{\nu}\right]\left[X_{\nu}, X_{\rho}\right]\left[X_{\rho}, X_{\mu}\right]\right)^{2}, \\
& \mathscr{P}_{10,2}(X)=\left(\frac{8}{3} \operatorname{Tr}\left[X_{\mu}, X_{\nu}\right]\left[X_{\nu}, X_{\rho}\right]\left[X_{\rho}, X_{\mu}\right]\right)^{4} .
\end{aligned}
$$

Remember that the Greek indices in the preceding four equations are summed from 1 to, respectively, 3,4,6,10. Already for $S U(3)$ and $D=3$, however, more ingenuity is required; using symbolic computation, we have found the result (the $d^{A B C}$ are the $S U(3)$ symmetric structure constants)

$$
\mathscr{P}_{3,3}(X)=\left(\frac{3}{16} f^{A B E} d^{C D E} f^{A^{\prime} B^{\prime} E^{\prime}} d^{C^{\prime} D^{\prime} E^{\prime}}-\frac{3}{10} f^{A B C} d^{D A^{\prime} E} f^{E B^{\prime} E^{\prime}} d^{E^{\prime} C^{\prime} D^{\prime}}\right) X_{\mu}^{A} X_{\mu}^{A^{\prime}} X_{\nu}^{B} X_{\nu}^{B^{\prime}} X_{\rho}^{C} X_{\rho}^{C^{\prime}} X_{\sigma}^{D} X_{\sigma}^{D^{\prime}}
$$

or, equivalently,

$$
\mathscr{P}_{3,3}(X)=-\frac{3}{4} \operatorname{Tr}\left[X_{\mu}, X_{\nu}\right]\left\{X_{\rho}, X_{\sigma}\right\} \operatorname{Tr}\left[X_{\mu}, X_{\nu}\right]\left\{X_{\rho}, X_{\sigma}\right\}+\frac{6}{5} \operatorname{Tr} X_{\mu}\left[X_{\nu}, X_{\rho}\right] \operatorname{Tr} X_{\mu}\left[\left\{X_{\nu}, X_{\sigma}\right\},\left\{X_{\rho}, X_{\sigma}\right\}\right] .
$$

For higher dimensions and yet larger values of $N$, we expect the Pfaffians to increase rapidly in complexity. Let us nevertheless collect the following general relations whose validity follows upon inspection of the explicit form of the matrices $\mathscr{M}_{D, N}$ given above. Namely, we have, for any $N$,

$$
\begin{aligned}
& \left.\mathscr{P}_{10, N}(X)\right|_{X_{7}=\ldots=X_{10}=0}=\left(\mathscr{P}_{6, N}(X)\right)^{2},\left.\quad \mathscr{P}_{6, N}(X)\right|_{X_{5}=X_{6}=0}=\left(\mathscr{P}_{4, N}(X)\right)^{2}, \\
& \left.\mathscr{P}_{4, N}(X)\right|_{X_{4}=0}=\left(\mathscr{P}_{3, N}(X)\right)^{2} .
\end{aligned}
$$

Of course, these relations are consistent with the explicit $S U(2)$ results quoted above. We emphasize, however, that relations of this type do not hold for generic configurations, as we have verified numerically. In addition, for $N \geq 3$ and $D \geq 6$, we have found configurations $X_{\mu}$ whose associated Pfaffians are negative. We also observe that $\mathscr{P}_{D, N}(X)$ vanishes whenever the matrices $\boldsymbol{X}_{\mu}$ are restricted to lie in the regularly embedded $s u(N-1)$ subalgebra of $s u(N)$. Finally, $\mathscr{P}_{D, N}(X)$ vanishes if only two $X_{\mu}$ 's are different from zero.

Returning to the integral (3), the first question to ask is whether or not it exists. The following naive argument seems to indicate that $\mathscr{Z}_{D, N}$ is divergent: Diagonalizing one of the matrices $X_{\mu}$ and restricting to the subspace where the off-diagonal elements of the other $D-1$ matrices are zero as well, we see that the diagonal components of all $D$ matrices do not appear in the action anymore. One might therefore suspect that the 
fluctuations along the diagonals cause the integral to diverge. Explicit calculations disprove this reasoning: it is shown in [2] that for the gauge group $S U(2)$ the exact values of the integral (3) are ${ }^{5}$

$$
\mathscr{Z}_{D, 2}=\sqrt{8 \pi} \times\left\{\begin{array}{cc}
0 & D=3 \\
\frac{1}{4} & D=4 \\
\frac{1}{4} & D=6 \\
\frac{5}{4} & D=10
\end{array} .\right.
$$

It is an instructive exercise to repeat the $S U(2)$ calculations for the case of no supersymmetry $(\mathscr{N}=0)$. Omitting the Pfaffian, one obtains

$$
\int \prod_{A=1}^{3} \prod_{\mu=1}^{D} \frac{d X_{\mu}^{A}}{\sqrt{2 \pi}} \exp \left[\frac{1}{2} \operatorname{Tr}\left[X_{\mu}, X_{\nu}\right]\left[X_{\mu}, X_{\nu}\right]\right]=\left\{\begin{array}{cc}
\infty & D \leq 4 \\
2^{-\frac{3}{4} D-1} \frac{\Gamma\left(\frac{D}{4}\right) \Gamma\left(\frac{D-2}{4}\right) \Gamma\left(\frac{D-4}{4}\right)}{\Gamma\left(\frac{D}{2}\right) \Gamma\left(\frac{D-1}{2}\right) \Gamma\left(\frac{D-2}{2}\right)} \quad D \geq 5
\end{array} .\right.
$$

One concludes that despite the valleys of the potential, entropic effects of the measure can become strong enough to overwhelm the possible divergences. Therefore we conjecture the integral $\mathscr{Z}_{D, N}$ to be finite for all $N$, even though no rigorous proof is available for $N \geq 3$ so far. There are two sources of such entropic effects. One is the increase in dimension with larger $D$ and $N$ : In the above example, the integral becomes convergent if only a sufficient (here: five) number of random matrices is added. Furthermore, we generally expect the integrals to become better behaved with larger $N$ since the relative weight of the valley configurations with regard to the generic configurations decreases like $N^{-1}$. Secondly, convergence is improved by supersymmetry because the Pfaffian damps the contribution of the valleys: It is easy to see that the Pfaffian vanishes if all $D$ matrices commute simultaneously.

Coming back to the supersymmetric case, we would like to find the generalization of Eq. (21) to higher rank gauge groups. Unfortunately the methods of [1,2] are very special to $S U(2)$ and cannot be generalized to $N>2$ in any obvious way. On the other hand, exploiting the suspected relationship of Eq. (3) to superstring instantons, Green and Gutperle [6] made a bold conjecture as to the value of the $D=10$ integral. Noticing, for $N=2$, a numerical coincidence between the result (21) and a term in their calculation of the D-instanton effective action, they suggested

$$
\mathscr{Z}_{D, N} \sim \sum_{m \mid N} \frac{1}{m^{2}} \quad \text { for } \quad D=10 .
$$

Here the sum runs over all divisors of $N$. If $N$ is prime, the sum is simply $1+\frac{1}{N^{2}}$. Now it is clear that such a conjecture is meaningless unless one very carefully specifies all the normalizations in Eq. (3)! Indeed, both the action and the polynomial $\mathscr{P}_{D, N}$ are homogeneous functions of the $X_{\mu}^{A}$, and therefore (3) is a pure number up to rescalings: It is impossible to introduce any non-trivial coupling constants. Fortunately, a natural normalization is suggested by the index calculations of [2]. An $N$-dependent "group factor" $\mathscr{F}_{N}$ has to be included, which

\footnotetext{
${ }^{5}$ While all the technical ingredients for this calculation can already be found in [1], the final result for the index obtained there differs from the correct result in Eq. (21) by a factor of 4. Also, that calculation did not take into account the deficit term of [2] needed for the interpretation as a Witten index.
} 
gives precisely the extra factor $\mathscr{F}_{2}=\sqrt{8 \pi}$ in (21). We can then extend the conjecture of [6] to also include the remaining dimensions $D=3,4,6$, and propose $^{6}$

$$
\mathscr{Z}_{D, N}=\mathscr{F}_{N} \times\left\{\begin{array}{cc}
0 & D=3 \\
\frac{1}{N^{2}} & D=4 \\
\frac{1}{N^{2}} & D=6 \\
\sum_{m \mid N} \frac{1}{m^{2}} & D=10
\end{array}\right.
$$

We note that the result $\mathscr{Z}_{3, N}=0$ for even $N$ is a trivial consequence of the reflection antisymmetry $X_{\mu} \rightarrow-X_{\mu}$; on the other hand, as our formulas (18), (19) for the $D=3, N=3$ Pfaffian shows, the result (if true!) is all but trivial for odd $N$.

It seems natural to assume that $\mathscr{F}_{N}$ is independent of the dimension. Extending the assumptions going into the index calculation of [2] to arbitrary $N \geq 2$ we find, with our normalizations of the integral (3),

$$
\mathscr{F}_{N}=\frac{2 \frac{N(N+1)}{2} \pi^{\frac{N-1}{2}}}{2 \sqrt{N} \prod_{i=1}^{N-1} i !} .
$$

It should be stressed that our extended conjecture (24), if true, furnishes an exact analytic solution of the IKKT matrix model [3]. Indeed, the partition function of the IKKT model is defined by summing up the $D=10$ $S U(N)$ partition functions, and we thus propose

$$
\mathscr{Z}_{\mathrm{IKKT}}(\beta)=\sum_{N=0}^{\infty} \mathscr{Z}_{10, N} e^{-\beta N},
$$

with $\mathscr{Z}_{10, N}$ given as in Eqs. (24), (25), and $\mathscr{Z}_{10,0}=\mathscr{Z}_{10,1}=1$. The weakest part in our proposal is the form (25) of $\mathscr{F}_{N}$ (see also our results below): Firstly, we might still have missed an $N$-dependent factor in Eq. (25), and secondly, the correct definition of the sum over $N$ might involve a yet unknown $N$-dependent constant $\mathscr{C}_{N}$ :

$$
\mathscr{Z}_{\mathrm{IKKT}}(\beta)=\sum_{N=0}^{\infty} \mathscr{C}_{N} \sum_{m \mid N} \frac{1}{m^{2}} e^{-\beta N} .
$$

It is interesting to observe that for $D=10$ the proposed form (24) of $\mathscr{Z}_{D, N}$ is non-analytic in $N$ : For any real number $c$ with $1 \leq c \leq \frac{\pi^{2}}{6}$ we can find a sequence $\left\{N_{\omega}\right\} \subset$ IN such that $\lim _{\omega \rightarrow \infty} \sum_{m \mid N_{\omega}} \frac{1}{m^{2}}=c$. Such a result, if true, would give an explicit example of a case where even the assumption of the existence of a large $N$ limit is simply wrong. On the other hand, it is intriguing for the future that summing over all values of $N$, as proposed

\footnotetext{
${ }^{6}$ This proposal slightly differs, for $D=4,6$ and non-prime values of $N$, from an earlier version of the present article. We thank I.K. Kostov for alerting us to the fact that the present form is indeed more natural.
} 
for the IKKT model, clearly reinstates analyticity (as long as $\mathscr{C}_{N}$ is analytic), since we can rewrite Eq. (27) quite generally as

$$
\mathscr{Z}_{\text {IKKT }}(\beta)=\sum_{m=1}^{\infty} \frac{1}{m^{2}} \sum_{k=1}^{\infty} \mathscr{C}_{m k} e^{-\beta m k} .
$$

Clearly these conjectures are very much in need of direct evidence from the actual integral (3). Analytical methods to treat this type of integral for $N>2$ are not available for the moment, and we have therefore concentrated on a numerical evaluation of the $S U(2)$ and $S U(3)$ partition functions using Monte Carlo methods, which, as we will show, need to be quite intricate in order to cope with the large variations of the integrand, provoked by the presence of the valleys.

\section{Monte Carlo approach}

In the following, to simplify notations, we write the integral Eq. (3) as

$$
\mathscr{Z}_{D, N}=\int_{V} d x z_{D, N}(x),
$$

where $d=D\left(N^{2}-1\right)$ is the total dimension of the integral, with $x=\left(x_{1}, x_{2}, \ldots, x_{d}\right)$. The volume $V$, for the moment, is $V=\mathrm{IR}^{d}$. For a Monte Carlo evaluation of widely varying integrals (cf [7]), we generally attempt to split off a positive importance sampling function $\pi(x): z_{D, N}(x)=\pi(x) \times \tilde{z}_{D, N}(x)$ such that

$$
\int_{V} d x z_{D, N}(x)=\int_{V} d x \pi(x) \tilde{z}_{D, N}(x) .
$$

A judicious choice of $\pi$ will permit us to preferentially generate points in the valleys. This will much reduce the statistical fluctuations: Suppose we are able to draw $M$ independent random configurations $x_{i}, i=1, \ldots, M$ distributed according to the probability $p(x) \sim \pi(x)$ with $V_{\pi}=\int_{V} d x \pi(x)$. We then have

$$
\mathscr{Z}_{D, N}=V_{\pi} \times \frac{1}{M} \sum_{i=1}^{M} \tilde{z}_{D, N}\left(x_{i}\right)+\mathscr{O}\left(\frac{\sqrt{\delta^{2} \mathscr{Z}_{D, N}}}{\sqrt{M}}\right),
$$

with

$$
\delta^{2} \mathscr{Z}_{D, N}=V_{\pi}^{2} \times \frac{1}{M} \sum_{i=1}^{M}\left(\tilde{z}_{D, N}\left(x_{i}\right)\right)^{2}-V_{\pi}^{2} \times\left(\frac{1}{M} \sum_{i=1}^{M} \tilde{z}_{D, N}\left(x_{i}\right)\right)^{2} .
$$

The unavoidable statistical error will therefore depend on the second moment

$$
\int_{V} d x \pi(x) \tilde{z}_{D, N}^{2}(x)
$$

In principle, the Monte Carlo computation is possible only with choices of $\pi$ for which the second moment (33) exists. It should also be realized that, as is evident from Eq. (31), we need to know $V_{\pi}$, the integral of $\pi$. If the latter is unknown, we are usually only able to evaluate expressions of the type

$$
\frac{\mathscr{Z}_{D, N}}{V_{\pi}}=\frac{\int d x \pi(x) \tilde{z}_{D, N}(x)}{\int d x \pi(x)} .
$$


We summarize these last remarks by stating that every approximation $\pi(x)$ to the integrand $z_{D, N}$ in Eq. (3) can serve as a useful starting point for very precise Monte Carlo evaluations of the integral - for arbitrarily high dimension $d$-provided that $\int_{V} d x \pi(x) \tilde{z}_{D, N}^{2}(x)$ exists, that $\left|z_{D, N}(x)\right| \sim \pi(x)$ everywhere and, lastly, that the integral of $\pi(x)$ be known.

As a first step towards the evaluation of $\mathscr{Z}_{D, N}$, we compactify $z_{D, N}$ onto the surface of the $d$-dimensional unit hypersphere $S^{d}$. To do this, we introduce polar coordinates, which we succinctly denote by $\left(x_{1}, \ldots, x_{d}\right)=$ $\left(\Omega_{d}, R\right)$. The action $\mathscr{S}$

$$
\mathscr{S}=-\frac{1}{2} \operatorname{Tr} \sum_{\mu=1, \nu=1}^{D}\left[X_{\mu}, X_{\nu}\right]\left[X_{\mu}, X_{\nu}\right]
$$

is homogeneous in $x$ :

$$
\mathscr{S}\left(\Omega_{d}, R\right)=\mathscr{S}\left(\Omega_{d}, 1\right) \times R^{4},
$$

as is the Pfaffian $\left(k=(D-2)\left(N^{2}-1\right)\right)$

$$
\mathscr{P}_{D, N}\left(\Omega_{d}, R\right)=\mathscr{P}_{D, N}\left(\Omega_{d}, 1\right) \times R^{k} .
$$

The $R$-integration can now be performed exactly, and we find

$$
\mathscr{Z}_{D, N}=\frac{\int \mathscr{D} \Omega_{d} z_{D, N}\left(\Omega_{d}\right)}{\int \mathscr{D} \Omega_{d}}
$$

with

$$
z_{D, N}\left(\Omega_{d}\right)=2^{\left(N^{2}-1\right) \frac{D-4}{2}-1} \frac{\Gamma\left(\left(N^{2}-1\right) \frac{D-1}{2}\right)}{\Gamma\left(\left(N^{2}-1\right) \frac{D}{2}\right)} \times \frac{\mathscr{P}_{D, N}\left(\Omega_{d}, 1\right)}{\left[\mathscr{S}\left(\Omega_{d}, 1\right)\right]^{\frac{D-1}{2}\left(N^{2}-1\right)}} .
$$

Without being obligatory, the compactification has nevertheless considerably smoothed the integrand, whose $R$-dependence was extremely strong. Eq. (39) means that we can evaluate the integral Eq. (3) as the average value of $z_{D, N}\left(\Omega_{d}\right)$ over randomly chosen points $\Omega_{d}$ on the unit sphere $S^{d}$. Such points can easily be generated from Gaussian random numbers [7] $x_{i}, i=1, \ldots, d$, projected onto $S^{d}$.

We now face an a priori formidable technical difficulty: One needs an efficient algorithm for evaluating $z_{D, N}\left(\Omega_{d}\right)$. However, it is well-known that Pfaffians belong to the enfants terribles of numerical analysis. Direct evaluation of Eq. (5) is impossible. This has to be compared to the case of determinants, where very powerful algorithms are available. There is a direct, well-known relationship between Pfaffians and determinant since one has $(\operatorname{Pf} \mathscr{M})^{2}=\operatorname{det} \mathscr{M}$. Taking the square-root, we can always find the Pfaffian up to a sign. Unfortunately, we have found that for $N>2$ the Pfaffians ${ }^{7}$ are not positive semi-definite! As discussed in detail earlier, the direct computation of the Pfaffian is possible in generality only for $D=4,6$. For $D=10$ more ingenuity is required, except for $S U(2)$, where a convenient representation is available, cf. Eq. (17). Using $S O(10)$ invariance, we have also found a way around the obstacle in the case of $D=10, S U(3)$ : cf Eqs. (12), (13). $D=10$ and $N \geq 4$ require new ideas, which will not be treated in this paper.

\footnotetext{
${ }^{7}$ An exception seems to be dimension $D=4$, where we conjecture the Pfaffian $\mathscr{P}_{4, N}$ to be positive semi-definite for all $N$.
} 
After computation of $z_{D, N}\left(\Omega_{d}\right)$, the evaluation of $\mathscr{Z}_{D, N}$ with the simple "direct sampling" algorithm is straightforward, as soon as the variance of the integral exists and is sufficiently small (cf Eq. (33)). This latter condition is far from innocent, as can be seen from the data in Table 1, where we present our results for $S U(2)$. We actually give the mean in units of $\sqrt{8 \pi}$, cf Eq. (21).

Indeed, we have been unable to compute the $D=4$ integral, because the variance of the integral does not exist in that case. Let us mention in passing that the (absolute) divergence of any integral $\int d x f(x)$ is best checked not by direct sampling as in Eqs. (31), (38), but by performing a separate Metropolis random walk, to be discussed below, with $\pi=|f|$. For the case of $f=z_{D=4, N=2}^{2}$, the Markov chain of this random walk quickly gets stuck in one valley, which proves the divergence of the second moment for all intents and purposes. For the integral itself the random walk finds its way back out of the valley (since the integral is finite), but it cannot be calculated by the present method. This extremely powerful method to ascertain the absolute convergence of smooth high-dimensional integrals plays no role in usual statistical physics applications, where the weight function $\pi(x)$ (Boltzmann weight or density matrix for classical and quantum applications, respectively) always assures convergence. With this simple method, clearcut answers can be obtained both for the absolute convergence of the integral itself and for the variance. In cases of doubt, the convergence check has to be done first since the relative weight of rare but strong fluctuations is difficult to assess by direct sampling.

Direct sampling of the integral, while successful for $D=6,10$ for $S U(2)$, as shown, has proven unfeasible for higher gauge groups. We thus need to consider importance sampling. The canonical way to proceed would be to use an analytically tractable approximation $\pi \sim\left|z_{D, N}\right|$ with known integral $V_{\pi}$. Such functions are unfortunately yet unknown to us. Short of this, we will present in the remainder of this paper an efficient algorithm which allows us to compute the ratio $\mathscr{Z}_{D^{>}, N} / \mathscr{Z}_{D^{<}, N}$ for any of the pairs $D^{\ll} D^{>}$of interest to us. The idea of the method is that for any configuration of matrices $X_{1}, \ldots, X_{D^{>}}$describing a "valley" configuration with large weight, we will be able to find subensembles of $D^{<}$matrices (among the above) describing a configuration with an important contribution to $\mathscr{Z}_{D^{<}, N}$.

To implement the idea, we first note that one condition in the above Eq. (38) was rather too restrictive. Because of our particular way of compactifying the integral, we may generate random points $\Omega_{d}<$ on the surface of the sphere $S^{d^{<}}$not only from projected Gaussian variables in $\mathrm{IR}^{d^{<}}$, but also from any other volume isotropic with respect to $S_{d}<$. Surprisingly, one perfectly correct way to generate random $\Omega_{d^{<}}$consists in taking $d^{<}$components of random vectors on $S_{d^{>}}$. Generalizing further, we write

$$
\pi\left(\Omega_{X_{1}, \ldots, X_{D}>}\right)=\frac{1}{\nu} \sum_{\{\sigma\}} z_{D^{<}, N}\left(\Omega_{X_{i_{1}}, \ldots, X_{i D}<}\right),
$$

where $\sigma=\left(i_{1}, \ldots, i_{D^{<}}\right)$denotes a combination of $D^{<}$indices out of $D^{>}$, and $\{\sigma\}$ the sum over all the combinations. The total number $\nu$ of possible combinations is

$$
\nu=\left(\begin{array}{l}
D^{>} \\
D^{<}
\end{array}\right)
$$

In an effort to be completely explicit, we specify that $\Omega_{X_{1}, \ldots, X_{D}>}$ and $\Omega_{X_{i_{1}}, \ldots, X_{i D}<}$ denote points on the surface of $S_{d^{>}}$and $S_{d^{<}}$, respectively.

Table 1

Data for SU(2), direct evaluation of integral

\begin{tabular}{rlll}
\hline$D$ & Samples & Mean & Error \\
\hline 4 & $4 \times 10^{12}$ & 0.242 & $\infty$ \\
6 & $2 \times 10^{12}$ & 0.250 & $<0.001$ \\
10 & $1 \times 10^{8}$ & 1.250 & $<0.001$ \\
\hline
\end{tabular}


Table 2

Data for $\mathrm{SU}(2)$, ratio of partition functions

\begin{tabular}{lllr}
\hline $\mathrm{D}^{>} / \mathrm{D}^{<}$ & Samples & Mean & Error \\
\hline $6 / 4$ & $6.0 \times 10^{7}$ & 1.00 & $<0.01$ \\
$10 / 4$ & $4.0 \times 10^{7}$ & 5.00 & 0.01 \\
$10 / 6$ & $3.8 \times 10^{7}$ & 5.01 & 0.01 \\
\hline
\end{tabular}

The important point is now that the average value of $\pi\left(\Omega_{X_{1}, \ldots, X_{D}>}\right)$ is equal to $\mathscr{Z}_{D^{<}, N}$. We have just transformed $\mathscr{Z}_{D^{<, N},}$ into an integral of the same dimension as $\mathscr{Z}_{D^{>}, N}$. The last step of our procedure consists in using the same set of $X_{1}, \ldots, X_{D^{>}}$for a simultaneous evaluation of both integrands:

$$
\frac{\mathscr{Z}_{D^{>}, N}}{\mathscr{Z}_{D^{<}, N}}=\frac{\int \mathscr{D} \Omega_{d^{>}} \pi\left(\Omega_{X_{1}, \ldots, X_{D}>}\right) \times z_{D^{>}, N}\left(\Omega_{X_{1}, \ldots, X_{D}>}\right) / \pi\left(\Omega_{X_{1}, \ldots, X_{D}>}\right)}{\int \mathscr{D} \Omega_{d^{>}} \pi\left(\Omega_{X_{1}, \ldots, X_{D}>}\right)} .
$$

In practice, we generate points $X_{1}, \ldots, X_{D^{>}}$according to $\pi\left(\Omega_{X_{1}, \ldots, X_{D}>}\right)$ with the Metropolis algorithm: starting from an initial configuration on the unit sphere $\Omega_{d^{>}}=\left(x_{1}, \ldots, x_{d^{>}}\right)$, we propose a simple local change of two of the coordinates, which conserves the norm of the total vector, to arrive at $\Omega_{d}^{\prime}>$. The proposed move is accepted with probability $\min \left(1, \pi\left(\Omega_{d^{>}}^{\prime}\right) / \pi\left(\Omega_{d^{>}}\right)\right.$) (for an elementary introduction cf, eg, [8]). We also have to cure the sign problem which is completely marginal for $D=4,6,10$. In fact, since $\pi$ in Eq. (40) is not always positive, we write

$$
\frac{\int \mathscr{D} \Omega_{d} \pi \times z / \pi}{\int \mathscr{D} \Omega_{d} \pi}=\frac{\int \mathscr{D} \Omega_{d}|\pi| \times z /|\pi|}{\int \mathscr{D} \Omega_{d}|\pi| \operatorname{sgn}(\pi)} ;
$$

$|\pi|$ is now a positive weight, which can be used for Monte Carlo sampling [9].

As a result, we obtain the ratio between two different partition functions. We now present our results on the ratios of the partition functions, both for $S U(2)$ (see Table 2), which serves as a test, and for $S U(3)$ (see Table 3 ). Obtaining these data required a few days on ten workstations at one of our institutes (AEI Potsdam).

For $S U(2)$, the exact values for the three ratios 6/4, 10/4, 10/6 (cf Eq. (21)) are, respectively, 1,5,5, which are reproduced. For $S U(3)$, the numerical computation leads to a novel, and very strong result: Here the ratios in question are to a surprisingly good precision 1,10,10, which beautifully agrees with (24) for $N=3$.

Unfortunately, the price to pay for coming up with a powerful algorithm was that we had to give up computing the integrals themselves. We have not attempted to test our conjecture Eq. (25) for the group factor $\mathscr{F}_{N}$ which appears in the proposed form (24) of the partition functions $\mathscr{Z}_{D, N}$. A direct computation of the integrals themselves belongs to the same class of Monte Carlo problems as free energy calculations, which are notoriously difficult. As mentioned before, it seems preferable to find a good analytical approximation to the

Table 3

Data for SU(3), ratio of partition functions

\begin{tabular}{llll}
\hline $\mathrm{D}^{>} / \mathrm{D}^{<}$ & Samples & Mean & Error \\
\hline $6 / 4$ & $4.7 \times 10^{7}$ & 0.99 & 0.01 \\
$10 / 4$ & $6.0 \times 10^{7}$ & 9.95 & 0.09 \\
$10 / 6$ & $4.0 \times 10^{7}$ & 9.98 & 0.02 \\
\hline
\end{tabular}


integrands with known integrals. On the other hand, we believe that the difficulties in the calculation of the $D=10$ Pfaffians can be overcome, and that our ratio test of the extended Green-Gutperle conjecture can be extended to gauge groups with $N \geq 4$. In any case, and independent on whether the extended conjecture will turn out to be correct, we feel we have already demonstrated the usefulness of numerical calculations in getting reliable information on these fascinating and intricate supersymmetric models.

\section{Note added}

We are now able to numerically compute the integrals for $S U(3), S U(4)$ and $S U(5)$ directly [10] and to confirm the corrected formula Eq. (24). Meanwhile we have also received the impressive paper [11], where under certain assumptions (i.e. contour prescriptions) the result for arbitrary $N$ (but without working out $\mathscr{F}_{N}$ ) is arrived at by analytical methods.

\section{Acknowledgements}

W.K., H.N. and M.S. thank, respectively, the AEI Potsdam, the LPT-ENS Paris and the LPS-ENS Paris for hospitality. This work was supported in part by the EU under Contract FMRX-CT96-0012.

\section{References}

[1] A.V. Smilga, Yad. Fiz. 42 (1985) 728; Nucl. Phys. B 266 (1986) 45; A.V. Smilga, Yad. Fiz. 43 (1986) 215.

[2] P. Yi, Nucl. Phys. B 505 (1997) 307, hep-th/9704098; S. Sethi, M. Stern, D-Brane Bound State Redux, hep-th/9705046.

[3] N. Ishibashi, H. Kawai, Y. Kitazawa, A. Tsuchiya, Nucl. Phys. B 498 (1997) 467, hep-th/9612115.

[4] H. Nicolai, Nucl. Phys. B 140 (1978) 294.

[5] T. Suyama, A. Tsuchiya, Exact Results in $N_{c}=2$ IIB Matrix Model, hep-th/9711073.

[6] M.B. Green, M. Gutperle, D-Particle Bound States and the D-Instanton Measure, hep-th/9711107.

[7] W.H. Press, S.A. Teukolsky, W.T. Vetterling, B.P. Flannery, Numerical Recipes, 2nd edition, Cambridge University Press, 1992.

[8] W. Krauth, Introduction To Monte Carlo Algorithms, in Advances in Computer Simulation, Lecture Notes in Physics, Springer Verlag, 1998, cond-mat/9612186.

[9] R. Blankenbecler, D.J. Scalapino, R.L. Sugar, Phys. Rev. D 24 (1981) 2278.

[10] W. Krauth, M. Staudacher, work in progress.

[11] G. Moore, N. Nekrasov, S. Shatashvili, D-particle bound states and generalized instantons, hep-th/9803265. 